Urologe 2011 · 50[Suppl 1]:163-164 DOI 10.1007/s00120-011-2691-7

Online publiziert: 26. August 2011

(c) Springer-Verlag 2011

\author{
J.A. Steffens ${ }^{1} \cdot 0$. W. Hakenberg ${ }^{2}$ \\ ${ }^{1}$ Klinik für Urologie und Kinderurologie, zertifiziertes Prostatakarzinom-Zentrum, \\ St. Antonius-Hospital Eschweiler, Eschweiler \\ ${ }^{2}$ Urologische Klinik und Poliklinik, Universitätsklinikum Rostock, Rostock
}

\title{
Positionspapier zur Urologischen Onkologie
}

spruch anderer Fachgebiete, die Onkologie als Ganzes und dann auch noch besser vertreten zu können, gemeinsam entgegentreten. Zweitens müssen wir innerhalb der Urologie die Arbeitsteilung und die Vernetzung zwischen ambulant und stationär, zwischen Niedergelassenem, Belegarzt und Klinik definieren und strukturieren. Dies sollte am besten geschehen, bevor andere es für uns tun.

Als Reaktion auf die politischen Vorgaben haben die Deutsche Gesellschaft für Urologie, der Berufsverband der Deutschen Urologen und die Arbeitsgemeinschaft „Urologische Onkologie“ (AUO) der Deutschen Krebsgesellschaft ein Positionspapier zur urologischen Onkologie erstellt, welches nachfolgend abgedruckt ist. Dieses Papier wurde als offener Brief allen gesundheitspolitischen Institutionen und berufspolitischen Standesvertretungen zugeleitet, ebenso wurde es allen Mitgliedern von DGU und BDU zugeschickt. Ein weiteres detailliertes Dokument zu den Anforderungskriterien der DKG-Zertifizierung für Onkologische Zentren wurde ebenfalls von DGU, BDU und AUO erarbeitet und der DKG zugesandt.

Es ist ein bescheidener Erfolg, DGU, BDU und AUO zu gemeinsamen Positionen zu bewegen. Denn die Sorge der Vertreter von niedergelassenen Urologen geht dahin, dass die Kliniken alles an sich ziehen wollen, die Sorge der onkologisch ausgerichteten Spezialisten besteht darin, dass der Widerstand der Niedergelassenen dazu führen wird, dass der gesundheitspolitische Zug mit der hämatoonkologischen Lokomotive abgefahren sein wird, bevor die Urologen sich zusammenfinden.

Wichtig ist, dass Letzteres verhindert wird, wenn es nicht schon zu spät ist. Es muss allen klar sein, dass es um die $\mathrm{Zu}$ kunft der Urologie geht und dass es damit um die Arbeit der Kollegen von morgen geht. Sektorale Befindlichkeiten und Besitzstandswahrung als politische Ziele werden nur dazu führen, dass andere Kräfte bestimmen. Deshalb müssen wir gemeinsam als deutsche Urologie auftreten.

So muss die Urologie die Kriterien zur Behandlung uroonkologischer Tumoren in Onkologischen Zentren maßgeblich selbst definieren und dies nicht gesundheitspolitischen Gremien überlassen. Gefordert wird in dem Positionspapier auch eine Neudefinition der Onkologievereinbarung, die ohnehin schon föderal völlig unkontrolliert ist und einer patientenschädlichen Indikationsausweitung Vorschub leistet.

\section{(2) Konkretisierung der uro- onkologischen Weiterbildung in der Weiterbildungsordnung}

Zusätzlich wird eine Neudefinition der uroonkologischen Weiterbildung in der Weiterbildungsordnung angestrebt. Dieser Punkt erregt bislang am meisten Kritik aus den Reihen besonders der niedergelassenen Kollegen, da dies vermeint-

Prof. Dr. J.A. Steffens ist Präsident der Deutschen Gesellschaft für Urologie e.V.

Prof. Dr. O. W. Hakenberg ist Generalsekretär der Deutschen Gesellschaft für Urologie e.V.
Zwei große Konfliktbereiche gilt es zu bearbeiten: Erstens müssen wir dem An- 
lich den Facharzt abwerten würde. Wie kaum ein anderes Fachgebiet wurde die Urologie durch Auslagerungen aus dem Facharzt in den letzten Jahren beschnitten (Andrologie, medikamentöse Tumortherapie, fachgebundenes Röntgen, Labor, Mikrobiologie). Die Musterweiterbildungsordnung wird vom Deutschen Ärztetag beschlossen, der mehrheitlich aus Hausärzten und Internisten besteht. Eine einfache Reintegration ist politisch gegenwärtig kaum durchsetzbar. Was das Positionspapier fordert, ist der „Parallelerwerb" für die medikamentöse Tumortherapie (d. h. während der Weiterbildungszeit zum Facharzt an Kliniken, in denen die Systemtherapie auch von der Urologie durchgeführt wird). Denn klar sein muss, dass die Kompetenz der Chemotherapie nur dann vorhanden sein kann, wenn sie auch erlernt wurde. Zusätzlich dazu soll die Möglichkeit der „Onkologischen Urologie" definiert werden [mit komplexer Tumorchirurgie und komplexer Systemtherapie (z. B. metastasiertes Blasenkarzinom, metastasierter Hodentumor, Sekundärtherapie)].

Da der Facharzt für Urologie allein nicht immer ausreichende Qualifikation für große tumorchirurgische Eingriffe und solche komplexen Systemtherapien bietet, soll unser onkologischer Schwerpunkt durch eine solche operativ und medikamentös geprägte 2-jährige Weiterbildung gefördert werden. Eine solche Schwerpunktbezeichnung wird niemanden, der heute niedergelassen ist, in seiner Tätigkeit tangieren, sondern soll das Fach besser positionieren, um interdisziplinär in den Kliniken auch in Zukunft onkologisch kompetent dastehen zu können.

Stittem

J.A. Steffens

0 .

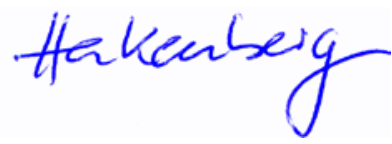

O.W. Hakenberg 